\title{
Analysis of the Impact Response to Backup Clamp from Broken Transmission Line Tension Clamp
}

\author{
Dengjie ZHU, ${ }^{\mathrm{a},}$, Yongli LIAO ${ }^{\mathrm{a}}$, Hao $\mathrm{LI}^{\mathrm{a}}$, Jie TANG ${ }^{\mathrm{b}}$ and Zhidu HUANG ${ }^{\mathrm{b}}$ \\ a Electric Power Research Institute, CSG, Guangzhou, Guangdong, China \\ ${ }^{\mathrm{b}}$ Electric Power Research Institute of Guangxi Power Grid Co.,Ltd.,Nanning, China
}

\begin{abstract}
The power company has improved the safety of important crossover lines by installing backup clamps to the tensile wire clamps. However, it remains to be studied whether the backup clamp can withstand the impact of the wire tension when it breaks. In this paper, the finite element model of the tension clamp with backup clamp is established and verified the accuracy of the model with an actual line as the engineering background. The results show that the back-up clamp force instantaneously reaches $49501.1 \mathrm{~N}$ at the moment of tension clamp breakage, and the effectiveness of the back-up clamp is indeed affected. Finally, the effect of the installation height on the impact coefficient is combined to give suggestions to improve the effectiveness of the backup clamp.
\end{abstract}

Keywords. Backup cable clamps, Impact Response, Life and death unit method, Effectiveness.

\section{Introduction}

The use of backup clamps, the original purpose is to prevent the combined effect of adverse factors such as ice, dance and sub-grade distance oscillation, resulting in damage to the fixture or the conductor from the tension clamps[1]. With the rapid extension of high-speed railroads, highways and other transportation networks across the country, some transmission lines in the suburbs originally did not belong to the important cross section, gradually transformed into cross-over lines. In order to improve the safety of these lines, the power grid company on these lines for centralized transformation, one of the important measures is the installation of backup line clamp. The installation of backup clamps can play a three-fold role: share the mechanical load of the wire tension clamp, increase the security margin of the tension clamp; additional diversion lines can play a role in shunting, reducing the failure rate of the tension clamp diversion plate heat; in extreme cases, once the tension clamp fracture failure, can prevent the wire from falling and continue to guide the flow.

The most common type of back-up clamps are pre-twisted back-up clamps. Figure 1 shows a pre-twisted backup clamp installed on a transmission line. The backup clamps consist of two main components. The system that provides mechanical tension consists

\footnotetext{
${ }^{1}$ Corresponding Author, Dengjie ZHU, Electric Power Research Institute, CSG, Guangzhou, Guangdong, China; Email: zhudj@csg.cn.
} 
of a set of safety backup clamps, a set of reinforcement splice strips, a section of strand filler strip, a set of thimbles and a set of shackles. The system providing electrical diversion consists of a set of shunt strips. The principle of operation of the backup clamps is that the pre-twisted wire legs are twisted together to form a hollow tube with a pre-formed ring at the rear, which is fixed to the connection fixture. The tubular structure formed by the legs of the pre-twisted wire is naturally wrapped around the conductor after installation to create an extremely strong grip.

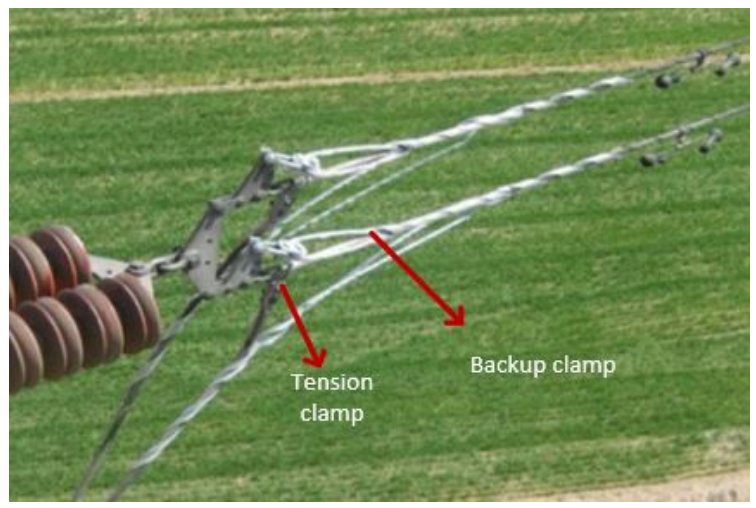

Figure 1. Pre-twisted backup clamp.

Most of the research on backup line clamps at home and abroad has focused on engineering applications. Xu Ying [2] through the analysis of various provinces and cities transmission line breakage accident cases, the technical characteristics of the backup clamps, advantages and disadvantages and the application in existing areas, the results show that the use of backup clamps can ensure the safe and stable operation of the power grid; Hu Guangya [3] proposed a carbon fiber composite core wire based on pre-twisted backup clamps protection crimping process improvement measures, the future of carbon fiber composite core wire and supporting gold Crimping construction has a positive guiding role; Huang Jinling [4] for the large wire diameter wire corrosion-resistant wire clamp fracture proposed the use of pre-twisted backup wire clamp measures.

This paper analyses the impact effect of the conductor on the backup clamps when the transmission line tension-resistant clamps are broken, firstly, a conductor-clamp finite element model with backup clamps is established for an actual line and the accuracy of the model is verified. A transient analysis of the model is carried out by using the raw-dead unit method to simulate the fracture of the tension-resistant wire clamp. The results show that at the moment of fracture, the axial force of the backup clamp reaches $49501.1 \mathrm{~N}$. The displacement in the $\mathrm{Y}$ and $\mathrm{Z}$ directions of the connection between the backup clamp and the conductor reaches $38.77 \mathrm{~cm}$ and 3.05 $\mathrm{cm}$, while no large displacement occurs in the X direction. The effect of the height of the backup clamp on the impact coefficient was also analysed and the results showed that the impact coefficient increased with the increase in the height of the backup clamp within a certain range. 


\section{Finite Element Model Building}

\subsection{Introduction to Transmission Lines}

A $500 \mathrm{kV}$ cross-over line as the engineering background, the cross-over file by a base tension tower, a linear tower and connecting the two towers of the conductor, insulator strings. The actual operation of the crossover section is shown in figure 2 . In order to improve the safety of the cross-over line, the power grid company added a pre-twisted backup wire clamp to the tension-resistant wire clamp.

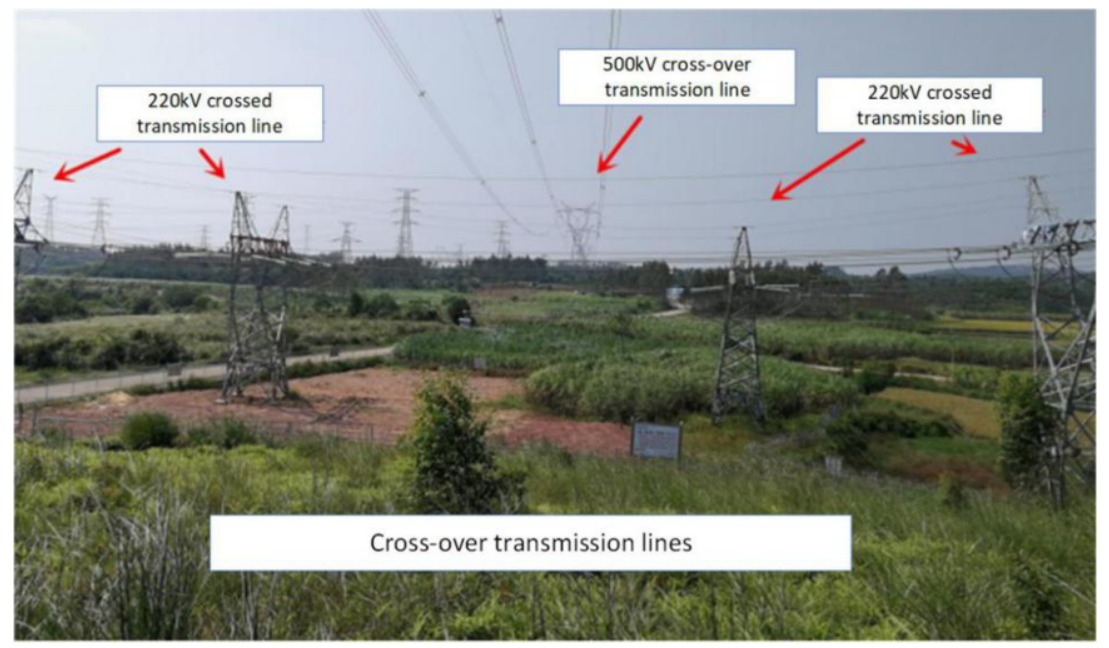

Figure 2. Site plan of $500 \mathrm{kV}$ crossover line.

\subsection{Finite Element Model}

In order to simplify the analysis and save calculation costs, the insulator strings and towers were not considered in the modelling. ANSYS finite element software was used to create a simplified finite element model of a sub-conductor of the right-phase conductor, its tensor clamps and backup clamps, with fixed end constraints at the ends of the conductor and at the hook points of the backup clamps. The key parameters of the conductor are listed in table 1, and the tensor clamps, conductor and backup clamps are modelled using LINK180 rod units.

Table 1. JL/LB20A-400/35 conductor specifications.

\begin{tabular}{ccccccc}
\hline $\begin{array}{c}\text { Wire } \\
\text { O.D./ } \\
\mathrm{mm}\end{array}$ & $\begin{array}{c}\text { Cross- } \\
\text { sectional } \\
\text { area/mm } / \mathrm{mm}^{2}\end{array}$ & $\begin{array}{c}\text { Modulus } \\
\text { of } \\
\text { elasticity/ } \\
\mathrm{GPa}\end{array}$ & $\begin{array}{c}\text { Calculated } \\
\text { mass } /(\mathrm{kg} \cdot \mathrm{k} \\
\left.\mathrm{m}^{-1}\right)\end{array}$ & $\begin{array}{c}\text { Calculated } \\
\text { pull-off } \\
\text { force } / \mathrm{kN}\end{array}$ & $\begin{array}{c}\text { Tensile } \\
\text { strength/ } \\
\mathrm{MPa}\end{array}$ & $\begin{array}{c}\text { Average } \\
\text { annual } \\
\text { running } \\
\text { tension } /(\mathrm{kN})\end{array}$ \\
\hline 26.82 & 425.24 & 66 & 1307.5 & 105.7 & 236.14 & 26.425 \\
\hline
\end{tabular}

As a highly flexible structure, the conductor can only withstand tensile forces and is prone to large displacement deformations when subjected to vertical loads, with a very high degree of non-linearity. As a typical suspension structure, the cables need to be shaped in order to determine the stresses and displacements of the cables in their 
initial state (under self-weight). After deformation, the cable structure has stresses and, due to the stress stiffening, has vertical stiffness, which allows various hydrostatic calculations to be carried out, therefore, deformation is an important step in the various calculations of the cable structure and is related to the accuracy of the calculations. The process of finding the shape of the conductor is based on the creation of a geometric model at the location of the chord of the transmission conductor and the setting of the real constants and actual parameters of the transmission conductor. Although the conductor tension is generated under load, a very small initial strain is defined at the moment 0 in order to obtain stability in the solution. Then, using the discharge horizontal stress of the transmission conductor in the actual project as the iterative target, a self-weight load is applied to obtain the horizontal stress in the finite element model. If the error with the discharge stress is greater than $1 / 100$, the deformation factor is defined to update the finite element model and the next iteration is performed. When the stress error is less than 1/100, the iteration is stopped and the static equilibrium state at this point is the initial configuration of the transmission conductor. The final finite element model of the conductor-withstanding wire clamp with backup clamp is shown in figure 3.

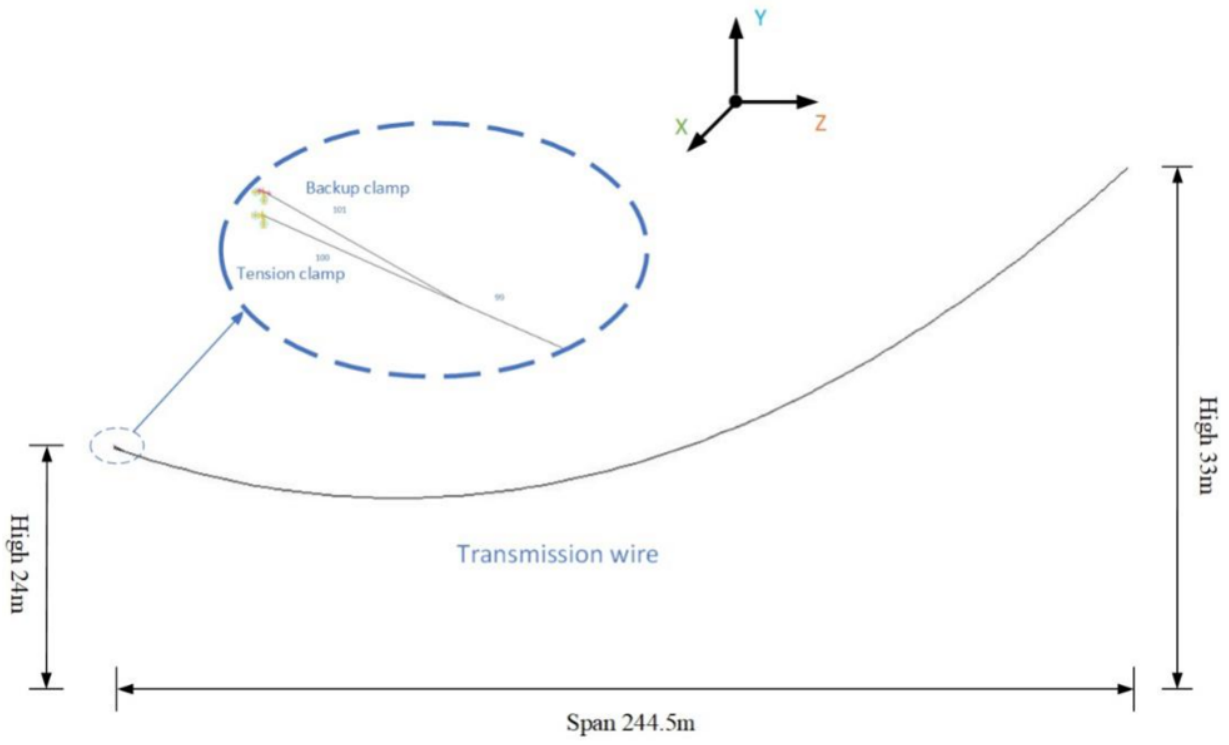

Figure 3. Finite element model of conductor-tensioner clamp with backup clamp.

\subsection{Model Validation}

In order to verify the correctness of the model built and to ensure the accuracy of the later analytical calculations, the arc sag values in the model are compared with the analytical solution of the wire arc sag for the purpose of verification. According to the literature [5], the approximate formula for calculating the central arc sag at unequal suspension point stall distances on overhead transmission lines is:

$$
f_{\frac{1}{2}}=\sqrt{1+\left(\frac{h}{L_{h=0}}\right)^{2}} \frac{\sigma_{0}}{\gamma}\left(\operatorname{ch} \frac{\gamma l}{2 \sigma_{0}}-1\right)
$$


where $\boldsymbol{h}$ is the height difference of the hanging point; $\boldsymbol{\sigma}_{\mathbf{0}}$ is the horizontal stress of the conductor; $\boldsymbol{\gamma}$ is the self-weight of the conductor than the load; $\boldsymbol{l}$ is the distance between the stalls; $\boldsymbol{L}_{\boldsymbol{h}=\mathbf{0}}$ indicates the length of the suspended chain line within the stalls of the overhead line at the same height suspension point, and the expression is:

$$
L_{h=0}=\frac{2 \sigma_{0}}{\gamma} \operatorname{sh} \frac{\gamma l}{2 \sigma_{0}}
$$

Through the formula (1), (2) calculation to get the conductor stall distance central arc dip analytical solution is $3.713 \mathrm{~m}$, and the model stall distance central arc dip is $3.800 \mathrm{~m}$, the error is $2.3 \%$. Compared with the analytical solution, the error of the model is smaller, which can indicate that the model meets the accuracy requirements.

\section{Backup Clamp Shock Response Analysis}

\subsection{Tension Clamp Fracture Simulation}

The impact effects of backup wire clamps are analysed using non-linear complete transient analysis. Tensioning clamp fracture is simulated using the following approach: firstly the whole system is allowed to equilibrate under the influence of gravity and the individual cells are made to generate internal forces. The sudden fracture of the tension binder is simulated using the raw and dead cell method in finite elements. The fracture of the tension binder is sudden and occurs within a very short period of time, so that the break time can be determined as $10^{-2} \mathrm{~s}$. In the whole time analysis, an automatic time step is used, with a time duration of $50 \mathrm{~s}$.

\subsection{Axial Force Response Analysis}

Using the above finite element model and the tension-resistant clamp fracture simulation method, the impact effect on the backup clamp after the tension-resistant clamp fracture is analysed. The extracted axial force time curve of the backup clamp is shown in figure 4 . It can be seen that at the beginning of the equilibrium phase, the backup clamp is almost force-free, and at the moment of the sudden fracture of the tensioning clamp at $5 \mathrm{~s}$, the axial force of the backup clamp instantaneously reaches 49501.1 N. After that, the wire and the backup clamp enter the non-linear free vibration phase under the action of damping. The tension on the original tensor clamps is transferred to the backup clamps. Eventually the whole system reaches a new equilibrium state. 


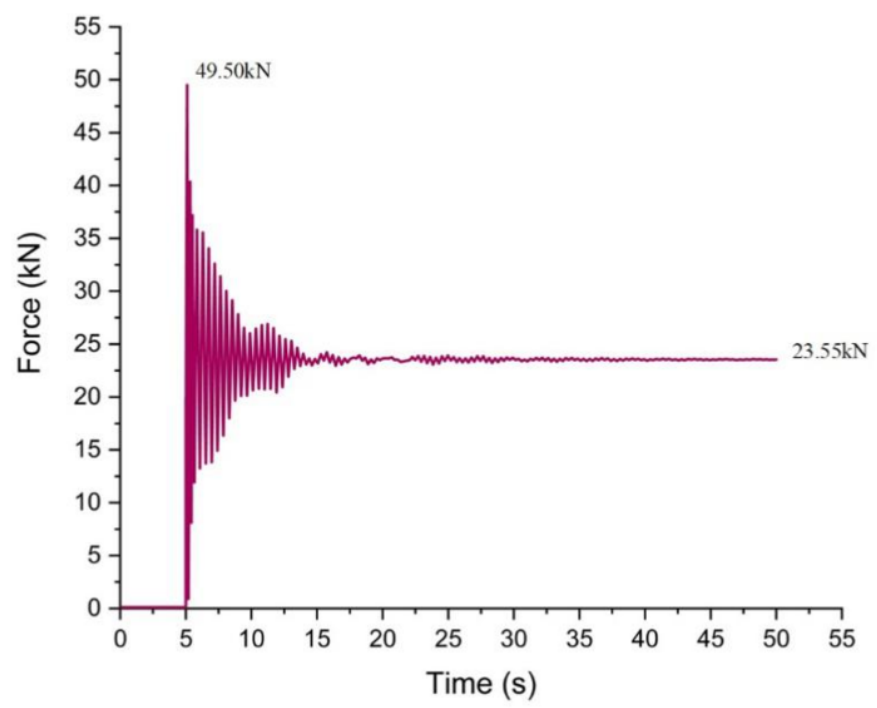

Figure 4. Backup clamp axial force time history curve.

\subsection{Displacement Response Analysis}

One end of the backup clamp is fixed to the upper end of the wire hook-up point and the other end is connected to the wire. The $\mathrm{X}, \mathrm{Y}$ and $\mathrm{Z}$ displacement curves for the $\mathrm{X}$, $\mathrm{Y}$ and $\mathrm{Z}$ directions were extracted from the end of the backup clamp connected to the wire as shown in figure 5. Backup wire clamp in the moment of the break occurs, under the action of the wire tension, its $\mathrm{Y}$ direction and $\mathrm{Z}$ direction have produced a large displacement, the extreme value of the displacement of $38.77 \mathrm{~cm}$ and $3.05 \mathrm{~cm}$, respectively, when the wire and backup wire clamp to re-equilibrium, backup wire clamp equilibrium position than before the break equilibrium position has changed, the $\mathrm{Y}$ direction and $\mathrm{Z}$ direction displacement change amount of $28.56 \mathrm{~cm}$ and $2.72 \mathrm{~cm}$, while the backup clamp in the entire analysis process in the $\mathrm{X}$ direction basically did not produce displacement, because the $\mathrm{X}$ direction is perpendicular to the direction of the wire, the backup clamp in the entire process basically will not produce displacement in the $\mathrm{X}$ direction, which is also in line with the actual situation. 


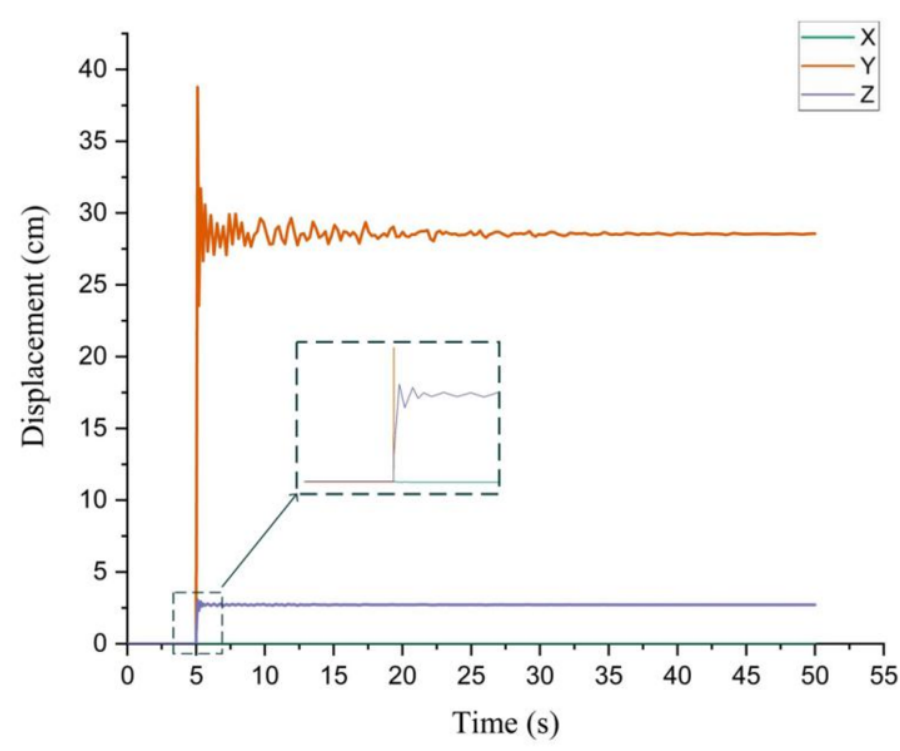

Figure 5. Time curve of displacement at the connection of the backup wire clamp to the wire.

\subsection{Influence of the Height of the Backup Wire Clamp Hanging Point on the Impact Factor}

The impact factor is the ratio of the response under dynamic impact loading to the static response. Also known as:

$$
\mu=R_{\max } / R_{s t c}
$$

$\boldsymbol{R}_{\boldsymbol{m a x}}$ is the maximum value of the force in the dynamic response; $\boldsymbol{R}_{\boldsymbol{s t c}}$ is the value of the force in static equilibrium. The above analysis shows the dynamic response of the backup clamp when the difference in height between the backup clamp hanging point and the tension-resistant clamp hanging point is $30 \mathrm{~cm}$. The impact factor can be calculated as 2.102 according to equation 3 . In order to study the effect of the height of the backup clamp on this impact response, the difference in height between the backup clamp hanging point and the tensioner clamp hanging point is set to $5 \mathrm{~cm}$, $10 \mathrm{~cm}, 15 \mathrm{~cm}, 20 \mathrm{~cm}, 25 \mathrm{~cm}$ and $30 \mathrm{~cm}$ respectively, and the impact response of the model is analysed by the previous method and the impact coefficient is calculated for different hanging point heights. The results are shown in figure 6 . 


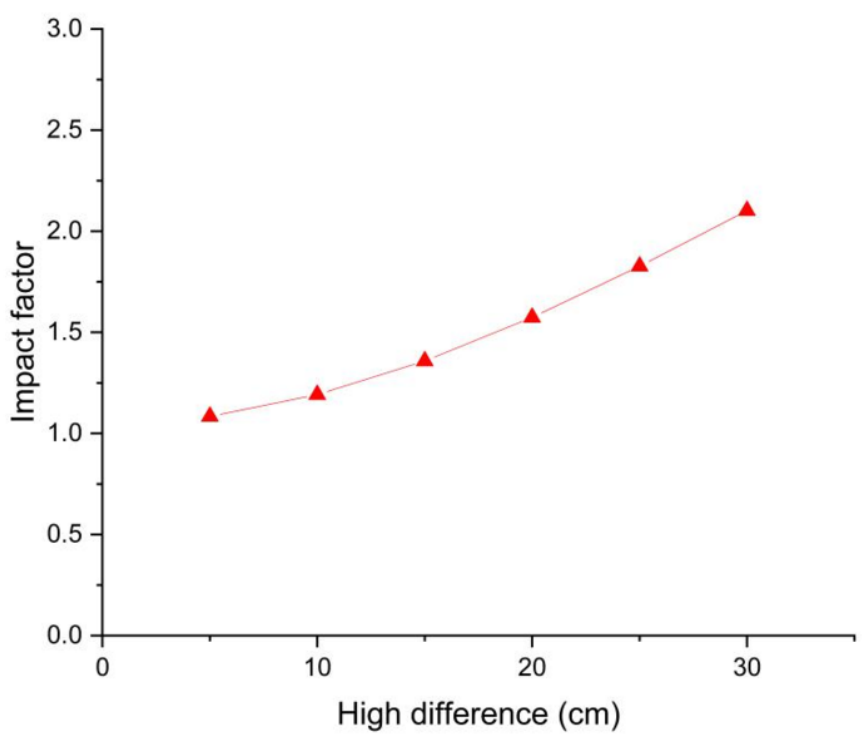

Figure 6. Impact coefficients for different height differences.

It can be seen that as the backup clamp hanging point gradually rises, the impact factor of the wire on the backup clamp impact when the tension-resistant clamp breaks is also gradually increasing. Therefore, in order to improve the effectiveness of the backup clamps, appropriate measures can be taken to reduce the height of the hanging point during installation.

\section{Conclusion}

This paper takes the transmission line backup clamp as the research object, through the non-linear complete transient analysis method, the backup clamp is subjected to the impact response from the wire at the time of the tensioning clamp breakage, combined with the above analysis results can be found.

(1) This paper uses an actual transmission line as the engineering background and establishes a finite element model of the conductor-tensioner clamp with a backup clamp, and verifies the accuracy of the model by comparing it with the analytical solution.

(2) The use of life and death of the unit method to simulate the fracture of the tension-resistant clamps, the backup clamps at the time of fracture transient analysis, the results show that at the moment of fracture the backup clamp axial force instantly reached $49501.1 \mathrm{~N}$, the backup clamp and the wire connection $\mathrm{Y}$ direction and $\mathrm{Z}$ direction displacement reached $38.77 \mathrm{~cm}$ and $3.05 \mathrm{~cm}$, while the $\mathrm{X}$ direction did not occur larger displacement.

(3) Analysis of the impact coefficient of the backup clamp hanging point height, the results show that in a certain range with the backup clamp installation height 
increase the impact coefficient is also greater. This conclusion also provides a reference basis for the installation height of the backup clamps in the project.

(4) This analysis does not consider the influence of transmission towers and insulator strings, so there may be some errors.

\section{Acknowledgments}

The authors would like to acknowledge the financial support provided for this research work by fund project: Science and Technology Project of Guangxi Power Grid Co.,Ltd. (GXKJXM20190616).

\section{References}

[1] Sun Zitang 2011 Research and progress of breeze vibration of anti-vibration hammer-transmission line system. J. Journal of Hefei University of Technology. 34 743-747

[2] Xu Ying and Zhao Huanqing 2018 Analysis of the application of safety backup wire clamps in Guangzhou area. J. Communication World.264-265

[3] Hu Guangya, Hu Shubing, Wu Hao and Sun Dongwang 2018 Improvement of crimping process of carbon fiber composite core conductor based on pre-twisted wire safety backup wire clamp protection. J. Jiangxi Electric Power.42 26-29

[4] Huang Jinling, Lin Junfa and Wei Pecai 2019 Large wire diameter conductor tension-resistant wire clamp rust fracture preventive measures to explore. J. Guangxi Electric Power.42 66-70

[5] Meng Suimin, Kong Wei and Tang Bo 2015 Design of overhead transmission lines (2nd ed.) (Beijing:China Electric Power Press) p 70 